\title{
Single Spin Detection with Entangled States
}

\author{
Hideaki Hakoshima* and Yuichiro Matsuzak \\ Nanoelectronics Research Institute National Institute of Advanced Industrial Science and Technology (AIST) \\ 1-1-1 Umezono, Tsukuba, Ibaraki 305-8568, Japan
}

\begin{abstract}
Single spin detection is one of the important tasks in the field of quantum metrology. Many experiments about the single spin detection has been performed. However, due to the weak magnetic fields from the single spin, a long measurement time is required to achieve a reasonably high signalto-noise ratio. Here, we propose an alternative way to realize rapid and accurate single spin detection with entangled states. While it is known that entanglement can improve the sensitivity to measure globally applied magnetic fields, we investigate a strategy to use the entanglement for detecting spatially inhomogeneous magnetic fields from the target single spin. We show that the entanglement significantly increases the signal to noise ratio for the single spin detection even under the effect of realistic noise. Our results pave the way for practical single spin detection.
\end{abstract}

\section{INTRODUCTION}

Magnetic field sensors with high sensitivity have been studied for many years due to the wide applications in various areas, such as biology and materials sciences. For the detection of the weak magnetic fields, many types of magnetic field sensors have been developed such as SQUID [1, 2], Hall sensors 3], and optomechanics [4.

Especially, a qubit-based sensor has been considered as an attractive approach to achieve a high sensitivity where a qubit is used as a probe of the magnetic field sensors. When the frequency of the qubit is shifted due to the external magnetic fields, Ramsey type measurements let us detect the amplitude of the magnetic fields. There are many experimental demonstrations along this direction such as neutral atoms (atomic vapor cells [5-7], cold atomic clouds [8-10]), trapped ions [11, 12], superconducting flux qubit [13 15, and nitrogen-vacancy centers in diamond [16, 17.

One of the ultimate goals of enhancing the sensitivity of magnetic sensors is an efficient detection of the single (electron or nuclear) spin [17, 18, and extensive research has been conducted, including both theoretical and experimental approaches 17 31. Since the single spin induces magnetic fields, a sensitive magnetic field sensor provides us with the way to detect the spin. However, the magnetic field from the single spin is typically weak, and this requires a long measurement time to compensate the low signal to noise ratio. So the improvement of the sensitivity is essential for the rapid and accurate spin detection.

On the other hand, it is known that, for the detection of global (spatially homogeneous) magnetic fields, an entanglement can enhance the performance of the qubitbased sensors. Especially, Greenberger-Horne-Zeilinger (GHZ) states can be used to outperform the standard quantum limit that classical sensors cannot surpass [12, 32, 42. There are many experimental demonstration

* hakoshima-hideaki@aist.go.jp to generate the GHZ states with several systems 12,43 45.

In this paper, we propose to use an entanglement of probe spins for the detection of the target single spin. We assume that the probe spins are two-level systems (qubits), and the frequency of the probe spins is affected by the magnetic fields from the target spin due to the dipole-dipole interactions. We consider using the GHZ states of the probe spins to detect the target spin. Although there are many previous researches about global magnetic field sensors with entanglement [12, 32,42, the single spin detection with the entangled probe spins has not been discussed. Importantly, due to the dipoledipole interaction between the target single spin and the probe spins, the entangled probes needs to detect the spatially inhomogeneous magnetic field. Especially, magnetic fields on the probe spins far from the target spin are much weaker than that of probe spins close to the target spin. Actually, for the single spin detection, the probe spins far from the target spin just induce noise without contributing the increase of the signal, and therefore the increase of the number of the probe spins does not necessarily improve the signal to noise ratio. This is stark contrast to the global magnetic field sensors with entangled probe spins where the signal to noise ratio is monotonically increased with increasing the number of the probe spins [12, 32, 42.

By considering these conditions, we investigate the performance of the single spin detection with entangled states where we optimize the number of the probe spins. Moreover, we compare the strategy to use the entangled probe spins with other strategies to use either a single probe spin or separable ensemble probe spins. We find that the single spin detection with the entangled probe spins achieves a few orders of magnitude better sensitivity than that with the single probe spin or the separable ensemble probe spins even under the effect of realistic noise.

The rest of this paper is organized as follows. In Sec. II, we introduce our setup of single spin detection with the GHZ states, and, we explain our measurement sequence with GHZ states. In Sec. III, we show the analytical and numerical solutions of the sensitivity to demonstrate the 
performance of the entangled probe spins. In Sec. IV, we summarize and conclude this paper.

\section{SETUP AND METHOD}

We explain the setup of our calculation. The configuration of the target spin and ensemble of probe spins is shown in the Fig. 1. There are $L$ probe spins homogeneously distributed with the density of $\rho$ inside a columnar substrate. The total Hamiltonian, which includes the dipole-dipole interaction between the target spin and the probe spins, is given by

$$
\begin{aligned}
\hat{H} & =\hat{H}_{\mathrm{T}}+\hat{H}_{\mathrm{P}}+\hat{H}_{\mathrm{I}} \\
\hat{H}_{\mathrm{T}} & =\frac{\omega^{(\mathrm{T})}}{2} \hat{\sigma}_{z}^{(\mathrm{T})} \\
\hat{H}_{\mathrm{P}} & =\sum_{j=1}^{L} \frac{\omega^{(\mathrm{P})}}{2} \hat{\sigma}_{z, j}^{(\mathrm{P})} \\
\hat{H}_{\mathrm{I}} & =G \sum_{j=1}^{L} \frac{\overrightarrow{\hat{\sigma}}^{(\mathrm{T})} \cdot \overrightarrow{\hat{\sigma}}_{j}^{(\mathrm{P})}-3\left(\overrightarrow{\hat{\sigma}}^{(\mathrm{T})} \cdot \frac{\vec{r}_{j}}{\left|\vec{r}_{j}\right|}\right)\left(\overrightarrow{\hat{\sigma}}_{j}^{(\mathrm{P})} \cdot \frac{\vec{r}_{j}}{\left|\vec{r}_{j}\right|}\right)}{\left|\vec{r}_{j}\right|^{3}},
\end{aligned}
$$

where $\omega^{(\mathrm{T})}\left(\omega^{(\mathrm{P})}\right)$ is a resonant frequency of the target (probe) spin, $G$ is a constant determined by the product of the magnetic moments of the target spin and the probe spins, and $\overrightarrow{\hat{\sigma}}_{j}^{(\mathrm{P})}=\left(\hat{\sigma}_{x, j}^{(\mathrm{P})}, \hat{\sigma}_{y, j}^{(\mathrm{P})}, \hat{\sigma}_{z, j}^{(\mathrm{P})}\right)$ is a set of the Pauli matrices of the probe spins at $\vec{r}_{j}=\left(x_{j}, y_{j}, z_{j}\right)$. We define the coordinate $(x, y, z)$ as shown in the Fig. 1 . Here, we assume a large detuning between the target spin and the probe spins $\omega^{(\mathrm{P})} \gg \omega^{(\mathrm{T})}$. In the rotating frame defined by the unitary transformation $\exp \left[i\left(\hat{H}_{\mathrm{T}}+\hat{H}_{\mathrm{P}}\right) t\right]$, we remove the terms oscillating with $\omega^{(\mathrm{T})}$ and $\omega^{(\mathrm{P})}$ (that is rotating wave approximation), and we obtain the effective Hamiltonian

$$
\hat{H}^{(\mathrm{eff})}=\sum_{j=1}^{L} \frac{G}{\left(r_{j}^{2}+z_{j}^{2}\right)^{3 / 2}}\left(1-\frac{3 z_{j}^{2}}{r_{j}^{2}+z_{j}^{2}}\right) \hat{\sigma}_{z}^{(\mathrm{T})} \hat{\sigma}_{z, j}^{(\mathrm{P})},
$$

where we set the cylindrical coordinate $r=\sqrt{x^{2}+y^{2}}$. Since we consider a case either the target spin is up or down, we replace $\hat{\sigma}_{z}^{(\mathrm{T})}$ with a classical value $s=1$ or -1 ;

$$
\hat{H}^{(\mathrm{eff})}=\sum_{j=1}^{L} \frac{\omega_{j}}{2} \hat{\sigma}_{z, j}^{(\mathrm{P})}
$$

where $\omega_{j}=\frac{2 G}{\left(r_{j}^{2}+z_{j}^{2}\right)^{3 / 2}}\left(1-\frac{3 z_{j}^{2}}{r_{j}^{2}+z_{j}^{2}}\right) s$. The variations of the distance between the target spin and each probe spin causes the inhomogeneous magnetic fields acting on the probe spins. We will consider either a single spin, an ensemble of separable spins [46, or entangled spins, as the probe states. When we consider the single probe spin, the probe spin is attached at the center bottom of

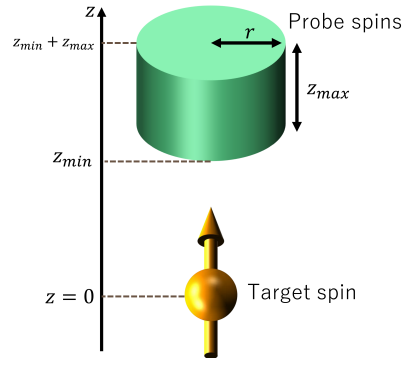

FIG. 1. This illustrates the positional relation between the target spin and the probe spins. The target spin is located at the origin of the coordinate, and the probe spins are homogeneously distributed with the density of $\rho$ inside a columnar substrate. The quantization axis of the probe spins and target spin are along the $z$ axis, and this system has a rotational symmetry around the $z$ axis.

the columnar substrate. As an entanglement, we choose the GHZ state:

$$
|\mathrm{GHZ}\rangle=\frac{1}{\sqrt{2}}(|\uparrow \cdots \uparrow\rangle+|\downarrow \cdots \downarrow\rangle)
$$

The time evolution of the quantum states under the effect of non-Markovian dephasing is described by the master equation

$$
\frac{\partial \hat{\rho}(t)}{\partial t}=i\left[\hat{\rho}(t), \hat{H}^{(\mathrm{eff})}\right]-\frac{t}{4 T_{2}^{2}} \sum_{j=1}^{L}\left[\hat{\sigma}_{z, j}^{(P)},\left[\hat{\sigma}_{z, j}^{(P)}, \hat{\rho}(t)\right]\right]
$$

where $\hat{\rho}(t)$ is the density operator at time $t$ and $T_{2}$ denotes the coherence time of the qubit. The first term of the right-hand side in (8) denotes the unitary time evolution of the Hamiltonian (6) and the second term denotes the effect of the dephasing from the environment.

We investigate three protocols for the single spin detection, which corresponds to the estimation of the value $s$ in this paper. The first one is to use a single probe spin for the detection of the target spin, as shown in Table I (i). The second one (ii) is to utilize an ensemble of separable probe spins to detect the target spin, which was theoretically studied in 46. The third one (iii) is to exploit the entangled probe spins to detect the target spin. We will show that the third protocol is much more efficient than the other two.

We describe the measurement sequence. Firstly, prepare an initial state of the probe spins (where the specific form of the initial state depends on the protocol, as shown in Table I). Secondly, let the quantum state evolve by Eq. (8) for a time $t$. Thirdly, measure the state by a specific readout basis (which also depends on the protocol as shown in Table I). Finally, we repeat 1-3 steps $N$ times. We assume that the state preparation time and readout time is negligibly small, and we can approximately obtain $N \simeq T / t$ where $T$ is a given total measurement time. 
TABLE I. Three protocols to estimate $s$

\begin{tabular}{|l||c|c|}
\hline Probe spins & Initital states & Readout basis \\
\hline \hline (i) Single & $|+\rangle$ & $(|\uparrow\rangle \pm i|\downarrow\rangle) / \sqrt{2}$ \\
(ii) Ensemble & $|++\cdots+\rangle$ & $\otimes_{j}[(|\uparrow\rangle \pm|\downarrow\rangle) / \sqrt{2}]$ \\
(separable) & $|+\cdots \uparrow\rangle \pm i|\downarrow \cdots \downarrow\rangle) / \sqrt{2}$ \\
(iii) Entangled & $|\mathrm{GHZ}\rangle$ & $(|\uparrow \cdots \uparrow\rangle \pm$ \\
\hline
\end{tabular}

\section{RESULTS}

For the first (i) and second protocol (ii), the sensitivity of $s$ to detect the single spin has been analyzed in [46. So, in this paper, we explain how to derive the sensitivity of $s$ with the third protocol (iii) where the probe spins are entangled. In the table II, we summarize the sensitivity of these three protocols for the comparison of our entanglement protocol with the other protocols.

We describe how we can derive the analytical form of the solution of the Eq. (8) as follows. $\quad \hat{\rho}(t)=\frac{1}{2}[|\uparrow \cdots \uparrow\rangle\langle\uparrow \cdots \uparrow|+| \downarrow \cdots \downarrow\rangle\langle\downarrow \cdots \downarrow|]+$ $\frac{e^{i \sum_{j} \omega_{j} t-L\left(\frac{t}{T_{2}}\right)^{2}}}{2}|\downarrow \cdots \downarrow\rangle\langle\uparrow \cdots \uparrow|+h . c$. , and we also obtain the expectation value $p=\operatorname{Tr}\left[\hat{P}_{\text {meas }} \hat{\rho}(t)\right]=\frac{1}{2} \pm$ $\frac{1}{2} e^{-L\left(\frac{t}{T_{2}}\right)^{2}} \sin \left(\sum_{j} \omega_{j} t\right) \simeq \frac{1}{2} \pm \frac{1}{2} e^{-L\left(\frac{t}{T_{2}}\right)^{2}}\left(\sum_{j} \omega_{j} t\right)$, where $\hat{P}_{\text {meas }}$ is the measurement operator of the readout basis as shown in Table I, and we assume the weak magnetic fields $\left|\sum_{j} \omega_{j} t\right| \ll 1$. In order to estimate the error of $s$, we calculate $\delta s:=\frac{\delta p}{\sqrt{N \mid}\left|\frac{\partial p}{\partial s}\right|}$, where $\delta p$ $(:=\sqrt{p(1-p)})$ is the standard deviation of $p$. To minimize the uncertainty, we choose $t=T_{2} / 2 \sqrt{L}$, and we obtain $\delta s=\frac{\sqrt{2} e^{1 / 4}}{\sqrt{T T_{2}}} \frac{L^{1 / 4}}{\left|\sum_{j} \frac{\partial \omega_{j}}{\partial s}\right|}$. Here, we take a continuous $\operatorname{limit}\left|\sum_{j} \frac{\partial \omega_{j}}{\partial s}\right| \simeq \rho\left|\iiint d x d y d z \frac{2 G}{\left(r_{j}^{2}+z_{j}^{2}\right)^{3 / 2}}\left(\frac{3 z_{j}^{2}}{r_{j}^{2}+z_{j}^{2}}-1\right)\right|=$ $4 \pi G \rho\left|\frac{z_{\max }}{\sqrt{r^{2}+z_{\max }^{2}}}-\frac{z_{\min }}{\sqrt{r^{2}+z_{\min }^{2}}}\right|$. This approximation is justified as $r, z_{\max }, z_{\min } \gg \rho^{-1 / 3}$, where $\rho^{-1 / 3}$ is the average distance among each probe spin. Moreover, we optimize the size of the columnar substrate (the number of the probe spins). Using $L=\rho \pi r^{2}\left(z_{\max }-z_{\min }\right)$, $\delta s=\frac{\sqrt{2} e^{1 / 4}}{4 G \pi^{3 / 4} \sqrt{T T_{2}}} \frac{z_{\min }^{3 / 4}}{\rho^{3 / 4}} \times f\left(\tilde{r}, \tilde{z}_{\max }\right)$, where $f\left(\tilde{r}, \tilde{z}_{\max }\right)=$ $\left[\tilde{r}^{2}\left(\tilde{z}_{\max }-1\right)\right]^{1 / 4} \times\left(\frac{\tilde{z}_{\max }}{\sqrt{\tilde{r}^{2}+\tilde{z}_{\max }^{2}}}-\frac{1}{\sqrt{\tilde{r}^{2}+1}}\right)^{-1}$, and $\tilde{r}=$ $r / z_{\min }, \tilde{z}_{\max }=z_{\max } / z_{\min }$. We numerically minimize $f\left(\tilde{r}, \tilde{z}_{\max }\right)=4.14$ as $\tilde{r}=1.87, \tilde{z}_{\max }=4.30$, and in this case, we obtain the number of the probe spins $L=\rho \pi \tilde{r}^{2}\left(\tilde{z}_{\max }-1\right) z_{\min }^{3}=35.9 \times \rho z_{\min }^{3}$.

We discuss our analytical results of the sensitivity. Firstly, we compare our result with the previous results (i) using the single probe spin or (ii) using separable ensemble probe spins for the single spin detection in the table II. Clearly, the scaling of $z_{\text {min }}$ of our scheme $\left(\delta s=O\left(z_{\text {min }}^{3 / 4}\right)\right)$ is different from those in the other two
TABLE II. Summary of results

\begin{tabular}{|c|c|c|}
\hline Probe spins & Global $B$ & Single spin detection \\
\hline (i) Single & $\delta B=O\left(L^{0}\right)$ & $\begin{array}{c}\delta s^{(\mathrm{s})}=\frac{\sqrt{2} e^{1 / 4}}{4 G \sqrt{T T_{2}}} z_{\min }^{3} \\
{[46}\end{array}$ \\
\hline $\begin{array}{l}\text { (ii) Ensemble } \\
\text { (separable) }\end{array}$ & $\begin{array}{l}\delta B=O\left(L^{-1 / 2}\right) \\
\text { (standard } \\
\text { quantum limit) }\end{array}$ & $\begin{array}{c}\delta s^{(\mathrm{sep})}=\frac{5.32 \sqrt{2} e^{1 / 4}}{4 G \sqrt{\pi} \sqrt{T T_{2}}} \frac{z_{\min }^{3 / 2}}{\sqrt{\rho}} \\
46\end{array}$ \\
\hline (iii) Entangled & $\begin{array}{c}\delta B=O\left(L^{-3 / 4}\right) \\
36,37,39\end{array}$ & $\begin{aligned} \delta s^{(\mathrm{en})}=\frac{4.14 \sqrt{2} e^{1 / 4}}{4 G \pi^{3 / 4} \sqrt{T T_{2}}} & \frac{z_{\min }^{3 / 4}}{\rho^{3 / 4}} \\
& \text { (our results) }\end{aligned}$ \\
\hline
\end{tabular}

schemes $\left(\delta s=O\left(z_{\text {min }}^{3}\right)\right.$ or $\left.\delta s=O\left(z_{\text {min }}^{3 / 2}\right)\right)$. These show that entanglement probe provides us with more efficient single spin detection than the other schemes especially when the target spin is located far from the probe spins. Secondly, we compare our results with the sensors to measure global magnetic fields $B$ with $L$ qubits in the table II. It is worth mentioning that, although we firstly analyze the performance to use the entangled states for the single spin detection, previous researches show that entangled states can enhance the performance of the global magnetic field sensors 12, 32, 42. From the table, we have found that the number of the qubits $L$ to measure the global magnetic fields corresponds to the density $\rho$ to detect the single spin. For example, the separable ensemble probe spins provide $\delta B=O\left(L^{-1 / 2}\right)$ or $\delta s=O\left(\rho^{-1 / 2}\right)$ (depending on the global magnetic field measurement or single spin detection) while the entangled probe spins provide $\delta B=O\left(L^{-3 / 4}\right)$ or $\delta s=O\left(\rho^{-3 / 4}\right)$. For the global magnetic field sensors with the entangled probe spins, we can just increase the number of the qubits to improve the signal to noise ratio. On the other hand, for the single spin detection, the sensitivity could be improved if we could increase the density while the other parameters were fixed. However, usually, the increase of the density degrades the coherence time $T_{2}$, and so it is not straightforward to increase just density with the same parameters, which is stark contrast with the global magnetic field sensing.

Here, we perform numerical simulations to evaluate the sensitivity to detect a single electron spin with realistic parameters. We consider the nitrogen vacancy (NV) centers as probe spins, which are one of the most promising systems to realize quantum enhanced sensors [16 30, 39, 47, 51]. In the Fig. 2, we plot $\delta s^{(\mathrm{s})} / \delta s^{(\mathrm{sep})}$ and $\delta s^{(\mathrm{s})} / \delta s^{(\mathrm{en})}$ against $z_{\min }$ where $\delta s^{(\mathrm{s})}, \delta s^{(\mathrm{sep})}$, and $\delta s^{(\mathrm{en})}$ denote the sensitivity of the single probe spin, separable ensemble probe spins, and entangled probe spins, respectively. These analytical form are shown in Table II. Here, for the numerical simulations, we choose $\rho=6.7 \times 10^{16} / \mathrm{cm}^{3}$ and $T_{2}=84 \mu$ s for an ensemble of the NV centers [16, 50, while we consider $T_{2}=2000 \mu$ s for a single NV center [51]. From this plot, we find that the ratio of the uncertainty is $\delta s^{(\mathrm{s})} / \delta s^{(\mathrm{en})} \simeq 500$ at $z_{\min }=1 \mu \mathrm{m}$, which means that the probe state with the entangled 


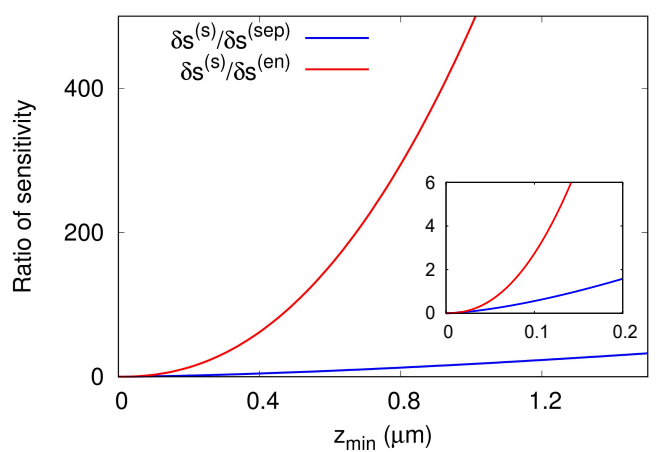

FIG. 2. Plot of the ratios of sensitivities $\delta s^{(\mathrm{s})} / \delta s^{(\mathrm{sep})}$ and $\delta s^{(\mathrm{s})} / \delta s^{(\mathrm{en})}$ against $z_{\min }$. Here, $\delta s^{(\mathrm{s})}, \delta s^{(\mathrm{sep})}$, and $\delta s^{(\mathrm{en})}$ denote the sensitivity of the single probe spin, separable ensemble probe spins, and entangled probe spins, respectively. We choose the typical parameters of NV centers, $\rho=6.7 \times 10^{16} / \mathrm{cm}^{3}$ and $T_{2}=84 \mu$ s for an ensemble of the NV centers, and $T_{2}=2000 \mu \mathrm{s}$ for a single NV center. The inset is a magnified view of the plot.

spins achieve 500 times higher sensitivity than that of the single spin. On the other hand, for the probe state with the separable spins, the ratio is $\delta s^{(\mathrm{s})} / \delta s^{(\mathrm{sep})} \simeq 17$ at $z_{\text {min }}=1 \mu \mathrm{m}$. These results show that the entangled probe spins achieves much higher sensitivity than that of the separable probe spins. Moreover, with the entangled probe spins, we have a wider parameter ranges about $z_{\text {min }}$ to beat the sensitivity of the single spin than with the separable probe spins. From the Fig. 2 (inset), we can see that $\delta s^{(\mathrm{s})} / \delta s^{(\mathrm{sep})}>1$ at $z_{\min }>0.15 \mu \mathrm{m}$, while $\delta s^{(\mathrm{s})} / \delta s^{(\mathrm{en})}>1$ at $z_{\min }>0.065 \mu \mathrm{m}$. The optimal number
$L$ of the probe spins with entangled states is proportional to $z_{\text {min }}^{3}$, and in the case of $z_{\text {min }}=1 \mu \mathrm{m}$, it is $L \simeq 2.4 \times 10^{6}$.

Furthermore, we numerically investigate the necessary time to detect a single spin. When the target spin is an electron spin, we can numerically calculate $\delta s$ with the parameters explained above, and $\delta s$ decreases as the total measurement time $T$ increases. We define the necessary time for the single spin detection as $T$ such that $\delta s=1$ is satisfied. Also, we introduce $T^{(\mathrm{s})}, T^{(\mathrm{s})}$, and $T^{(\mathrm{en})}$ to denote the necessary time for the single spin detection when the probe is a single spin, a separable ensemble, and an entangled state, respectively. We obtain $T^{(\mathrm{s})} \sim$ $10^{4} \mathrm{~s}, T^{(\mathrm{sep})} \sim 10^{2} \mathrm{~s}$, and $T^{(\mathrm{en})} \simeq 0.1 \mathrm{~s}$ at $z_{\min }=1 \mu \mathrm{m}$. Therefore, the entangled probe provides us with a much more rapid detection of the single target spin.

\section{SUMMARY AND CONCLUSION}

In conclusion, we have shown that entangled probe spins enable us to detect the target single spin with realistic parameters much more efficiently than the single probe spin or the separable ensemble probe spins. We expect that by using entangled states, more efficient detection of single spin will be achieved experimentally near the future.

\section{ACKNOWLEDGMENTS}

We are grateful to Syuhei Uesugi, S. Endo, and Junko Hayase for their assistant in this study. This work was supported by Leading Initiative for Excellent Young Researchers MEXT Japan, and is partially supported by MEXT KAKENHI (Grant No. 15H05870).
[1] RC Jaklevic, J Lambe, JE Mercereau, and AH Silver. Macroscopic quantum interference in superconductors. Physical Review, 140(5A):A1628, 1965.

[2] RL Fagaly. Superconducting quantum interference device instruments and applications. Review of scientific instruments, 77(10):101101, 2006.

[3] GD Howells, A Oral, SJ Bending, SR Andrews, PT Squire, P Rice, A De Lozanne, JAC Bland, I Kaya, and M Henini. Scanning Hall probe microscopy of ferromagnetic structures. Journal of magnetism and magnetic materials, 196:917-919, 1999.

[4] Stefan Forstner, Eoin Sheridan, Joachim Knittel, Christopher L Humphreys, George A Brawley, Halina Rubinsztein-Dunlop, and Warwick P Bowen. Ultrasensitive optomechanical magnetometry. Advanced Materials, 26(36):6348-6353, 2014.

[5] IK Kominis, TW Kornack, JC Allred, and Michael V Romalis. A subfemtotesla multichannel atomic magnetometer. Nature, 422(6932):596, 2003.

[6] Thomas Fernholz, Hanna Krauter, Kasper Jensen, Jacob Friis Sherson, Anders Søndberg Sørensen, and Eu- gene Simon Polzik. Spin squeezing of atomic ensembles via nuclear-electronic spin entanglement. Physical review letters, 101(7):073601, 2008.

[7] Wojciech Wasilewski, Kasper Jensen, Hanna Krauter, Jelmer Jan Renema, MV Balabas, and Eugene Simon Polzik. Quantum noise limited and entanglement-assisted magnetometry. Physical Review Letters, 104(13):133601, 2010.

[8] M Vengalattore, JM Higbie, SR Leslie, J Guzman, LE Sadler, and DM Stamper-Kurn. High-resolution magnetometry with a spinor Bose-Einstein condensate. Physical review letters, 98(20):200801, 2007.

[9] RJ Sewell, M Koschorreck, M Napolitano, B Dubost, $\mathrm{N}$ Behbood, and MW Mitchell. Magnetic sensitivity beyond the projection noise limit by spin squeezing. Physical review letters, 109(25):253605, 2012.

[10] Caspar F Ockeloen, Roman Schmied, Max F Riedel, and Philipp Treutlein. Quantum metrology with a scanning probe atom interferometer. Physical review letters, 111(14):143001, 2013. 
[11] I Baumgart, J-M Cai, A Retzker, Martin B Plenio, and Ch Wunderlich. Ultrasensitive magnetometer using a single atom. Physical review letters, 116(24):240801, 2016.

[12] D Leibfried, Murray D Barrett, T Schaetz, J Britton, J Chiaverini, Wayne M Itano, John D Jost, Christopher Langer, and David J Wineland. Toward Heisenberglimited spectroscopy with multiparticle entangled states. Science, 304(5676):1476-1478, 2004.

[13] Mustafa Bal, Chunqing Deng, Jean-Luc Orgiazzi, FR Ong, and Adrian Lupascu. Ultrasensitive magnetic field detection using a single artificial atom. Nature communications, 3:1324, 2012.

[14] Hiraku Toida, Yuichiro Matsuzaki, Kosuke Kakuyanagi, Xiaobo Zhu, William J Munro, Hiroshi Yamaguchi, and Shiro Saito. Electron paramagnetic resonance spectroscopy using a single artificial atom. Communications Physics, 2(1):33, 2019.

[15] Koichiro Miyanishi, Yuichiro Matsuzaki, Hiraku Toida, Kosuke Kakuyanagi, Makoto Negoro, Masahiro Kitagawa, and Shiro Saito. Nuclear magnetic resonance spectroscopy with a superconducting flux qubit. arXiv preprint arXiv:1906.07386, 2019.

[16] Thomas Wolf, Philipp Neumann, Kazuo Nakamura, Hitoshi Sumiya, Takeshi Ohshima, Junichi Isoya, and Jörg Wrachtrup. Subpicotesla diamond magnetometry. Physical Review X, 5(4):041001, 2015.

[17] JM Taylor, P Cappellaro, L Childress, L Jiang, D Budker, PR Hemmer, A Yacoby, R Walsworth, and MD Lukin. High-sensitivity diamond magnetometer with nanoscale resolution. Nature Physics, 4(10):810, 2008.

[18] Christian Degen. Nanoscale magnetometry: Microscopy with single spins. Nature nanotechnology, 3(11):643, 2008.

[19] JR Maze, Paul L Stanwix, JS Hodges, Sungkun Hong, JM Taylor, P Cappellaro, L Jiang, MV Gurudev Dutt, E Togan, AS Zibrov, et al. Nanoscale magnetic sensing with an individual electronic spin in diamond. Nature, 455(7213):644, 2008.

[20] Gopalakrishnan Balasubramanian, IY Chan, Roman Kolesov, Mohannad Al-Hmoud, Julia Tisler, Chang Shin, Changdong Kim, Aleksander Wojcik, Philip R Hemmer, Anke Krueger, et al. Nanoscale imaging magnetometry with diamond spins under ambient conditions. Nature, 455(7213):648, 2008.

[21] Marcus Schaffry, Erik M Gauger, John JL Morton, and Simon C Benjamin. Proposed spin amplification for magnetic sensors employing crystal defects. Physical review letters, 107(20):207210, 2011.

[22] C Müller, X Kong, J-M Cai, K Melentijević, A Stacey, M Markham, D Twitchen, J Isoya, S Pezzagna, J Meijer, et al. Nuclear magnetic resonance spectroscopy with single spin sensitivity. Nature communications, 5:4703, 2014.

[23] Tobias Staudacher, Fazhan Shi, S Pezzagna, Jan Meijer, Jiangfeng Du, Carlos A Meriles, Friedemann Reinhard, and Joerg Wrachtrup. Nuclear magnetic resonance spectroscopy on a (5-nanometer) 3 sample volume. Science, 339(6119):561-563, 2013.

[24] HJ Mamin, M Kim, MH Sherwood, CT Rettner, K Ohno, DD Awschalom, and D Rugar. Nanoscale nuclear magnetic resonance with a nitrogen-vacancy spin sensor. Science, 339(6119):557-560, 2013.

[25] K Ohashi, T Rosskopf, H Watanabe, M Loretz, Y Tao, $\mathrm{R}$ Hauert, $\mathrm{S}$ Tomizawa, $\mathrm{T}$ Ishikawa, J Ishi-Hayase,
S Shikata, et al. Negatively charged nitrogen-vacancy centers in a $5 \mathrm{~nm}$ thin $12 \mathrm{c}$ diamond film. Nano letters, 13(10):4733-4738, 2013.

[26] D Rugar, HJ Mamin, MH Sherwood, M Kim, CT Rettner, K Ohno, and DD Awschalom. Proton magnetic resonance imaging using a nitrogen-vacancy spin sensor. Nature nanotechnology, 10(2):120, 2015.

[27] Igor Lovchinsky, AO Sushkov, E Urbach, Nathalie P de Leon, Soonwon Choi, Kristiaan De Greve, R Evans, R Gertner, E Bersin, C Müller, et al. Nuclear magnetic resonance detection and spectroscopy of single proteins using quantum logic. Science, 351(6275):836-841, 2016.

[28] Nan Zhao, Jan Honert, Bernhard Schmid, Michael Klas, Junichi Isoya, Matthew Markham, Daniel Twitchen, Fedor Jelezko, Ren-Bao Liu, Helmut Fedder, et al. Sensing single remote nuclear spins. Nature nanotechnology, 7(10):657, 2012.

[29] Fazhan Shi, Qi Zhang, Pengfei Wang, Hongbin Sun, Jiarong Wang, Xing Rong, Ming Chen, Chenyong Ju, Friedemann Reinhard, Hongwei Chen, et al. Singleprotein spin resonance spectroscopy under ambient conditions. Science, 347(6226):1135-1138, 2015.

[30] Eisuke Abe and Kento Sasaki. Tutorial: Magnetic resonance with nitrogen-vacancy centers in diamondmicrowave engineering, materials science, and magnetometry. Journal of Applied Physics, 123(16):161101, 2018.

[31] Daniel Rugar, Raffi Budakian, HJ Mamin, and BW Chui. Single spin detection by magnetic resonance force microscopy. Nature, 430(6997):329, 2004.

[32] Vittorio Giovannetti, Seth Lloyd, and Lorenzo Maccone. Quantum-enhanced measurements: beating the standard quantum limit. Science, 306(5700):1330-1336, 2004.

[33] Luca Pezzé and Augusto Smerzi. Mach-Zehnder interferometry at the heisenberg limit with coherent and squeezed-vacuum light. Physical review letters, 100(7):073601, 2008.

[34] Vittorio Giovannetti, Seth Lloyd, and Lorenzo Maccone. Advances in quantum metrology. Nature photonics, $5(4): 222,2011$.

[35] Jonathan A Jones, Steven D Karlen, Joseph Fitzsimons, Arzhang Ardavan, Simon C Benjamin, G Andrew D Briggs, and John JL Morton. Magnetic field sensing beyond the standard quantum limit using 10-spin NOON states. Science, 324(5931):1166-1168, 2009.

[36] Yuichiro Matsuzaki, Simon C Benjamin, and Joseph Fitzsimons. Magnetic field sensing beyond the standard quantum limit under the effect of decoherence. Physical Review A, 84(1):012103, 2011.

[37] Alex W Chin, Susana F Huelga, and Martin B Plenio. Quantum metrology in non-Markovian environments. Physical review letters, 109(23):233601, 2012.

[38] Katarzyna Macieszczak. Zeno limit in frequency estimation with non-markovian environments. Physical Review A, 92(1):010102, 2015.

[39] Tohru Tanaka, Paul Knott, Yuichiro Matsuzaki, Shane Dooley, Hiroshi Yamaguchi, William J Munro, and Shiro Saito. Proposed robust entanglement-based magnetic field sensor beyond the standard quantum limit. Physical review letters, 115(17):170801, 2015.

[40] Shane Dooley, William J Munro, and Kae Nemoto. Quantum metrology including state preparation and readout times. Physical Review A, 94(5):052320, 2016. 
[41] Luca Pezzè, Augusto Smerzi, Markus K Oberthaler, Roman Schmied, and Philipp Treutlein. Quantum metrology with nonclassical states of atomic ensembles. Reviews of Modern Physics, 90(3):035005, 2018.

[42] Yuichiro Matsuzaki, Simon Benjamin, Shojun Nakayama, Shiro Saito, and William J Munro. Quantum metrology beyond the classical limit under the effect of dephasing. Physical review letters, 120(14):140501, 2018.

[43] Dietrich Leibfried, Emanuel Knill, Signe Seidelin, Joe Britton, R Brad Blakestad, John Chiaverini, David B Hume, Wayne M Itano, John D Jost, Christopher Langer, et al. Creation of a six-atom Schrödinger catstate. $\mathrm{Na}$ ture, 438(7068):639, 2005.

[44] Chao Song, Kai Xu, Hekang Li, Yu-Ran Zhang, Xu Zhang, Wuxin Liu, Qiujiang Guo, Zhen Wang, Wenhui Ren, Jie Hao, et al. Generation of multicomponent atomic Schrödinger cat states of up to 20 qubits. Science, 365(6453):574-577, 2019.

[45] Ahmed Omran, Harry Levine, Alexander Keesling, Giulia Semeghini, Tout T Wang, Sepehr Ebadi, Hannes Bernien, Alexander S Zibrov, Hannes Pichler, Soonwon Choi, et al. Generation and manipulation of Schr $\ "$ odinger cat states in Rydberg atom arrays. arXiv preprint arXiv:1905.05721, 2019.

[46] Syuhei Uesugi, Yuichiro Matsuzaki, Suguru Endo, Shiro Saito, and Junko Ishi-Hayase. Single spin detection with an ensemble of probe spins. arXiv preprint arXiv:1707.09824, 2017.

[47] CL Degen. Scanning magnetic field microscope with a diamond single-spin sensor. Applied Physics Letters, 92(24):243111, 2008.

[48] Emre Togan, Yiwen Chu, AS Trifonov, Liang Jiang, Jeronimo Maze, Lilian Childress, MV Gurudev Dutt, Anders Søndberg Sørensen, PR Hemmer, Alexander S Zibrov, et al. Quantum entanglement between an optical photon and a solid-state spin qubit. Nature, 466(7307):730, 2010.

[49] Loïc Rondin, Jean-Philippe Tetienne, Thomas Hingant, Jean-François Roch, Patrick Maletinsky, and Vincent Jacques. Magnetometry with nitrogen-vacancy defects in diamond. Reports on progress in physics, 77(5):056503, 2014.

[50] C Grezes, B Julsgaard, Y Kubo, WL Ma, M Stern, A Bienfait, K Nakamura, J Isoya, S Onoda, T Ohshima, et al. Storage and retrieval of microwave fields at the singlephoton level in a spin ensemble. Physical Review A, 92(2):020301, 2015.

[51] Gopalakrishnan Balasubramanian, Philipp Neumann, Daniel Twitchen, Matthew Markham, Roman Kolesov, Norikazu Mizuochi, Junichi Isoya, Jocelyn Achard, Johannes Beck, Julia Tissler, et al. Ultralong spin coherence time in isotopically engineered diamond. Nature materials, 8(5):383, 2009. 